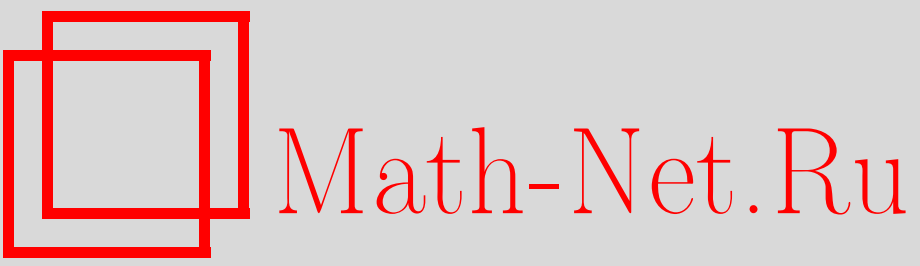

Е.И. Деза, К вопросу об обобщенных дискретных метрических структурах, Итоги науки и техн. Сер. Соврем. мат. и ее прил. Темат. обз., 2020, том 181, 22-29

DOI: https://doi.org/10.36535/0233-6723-2020-181-22-29

Использование Общероссийского математического портала Math-Net.Ru подразумевает, что вы прочитали и согласны с пользовательским соглашением

http://www. mathnet.ru/rus/agreement

Параметры загрузки :

IP: 54.197 .130 .99

26 апреля 2023 г., 04:39:24 




ИТОГИ НАУКИ И ТЕХНИКИ.

Современная математика и ее приложения.

Тематические обзоры.

Том 181 (2020). С. 22-29

DOI: $10.36535 / 0233-6723-2020-181-22-29$

УДК 519.1

\title{
К ВОПРОСУ ОБ ОБОБЩЕННЫХ ДИСКРЕТНЫХ МЕТРИЧЕСКИХ СТРУКТУРАХ
}

\author{
(c) 2020 г. $\quad$ Е. И. ДЕЗА
}

\begin{abstract}
АннотАция. В статье рассмотрены вопросы, связанные с построением и исследованием конусов полуметрик, квазиполуметрик (которые являются ориентированными аналогами симметричного случая полуметрик), и $m$-полуметрик (которые являются многомерными аналогами двумерного случая полуметрик).
\end{abstract}

Ключевые слова: расстояние, полуметрика, метрика, квазиполуметрика, многомерная полуметрика, разрез, мультиразрез, конус обобщенных дискретных метрических структур.

\section{ON GENERALIZED DISCRETE METRIC STRUCTURES}

\author{
(C) 2020 E. I. DEZA
}

\begin{abstract}
In this paper, we discuss problems related to construction and investigation of cones of semimetrics, quasi-semimetrics (which are oriented analogs of symmetric semimetrics), and $m$ semimetrics (which are multidimensional analogs of two-dimensional semimetrics).
\end{abstract}

Keywords and phrases: distance, semimetric, metric, quasi-semimetric, multidimensional semimetric, cut, multi-cut, cone of generalized discrete metric structures.

AMS Subject Classification: 54E25, 54E35

1. Введение. Понятие расстояния является одним из основных на только в математике и науке в целом, но и во всем человеческом бытии. Для обычной жизни расстояние представляет собой прежде всего оценку степени близости двух объектов или идей. В то же время термин метрика используется, как правило, для обозначения стандартного понятия меры (измерения).

Математические понятия метрики на множестве $X$ (т.е. функции $d(x, y)$ из $X \times X$ в $\mathbb{R}$, удовлетворяющей условиям $d(x, y) \geqslant 0$ с равенством только при $x=y, d(x, y)=d(y, x)$, и $d(x, y) \leqslant d(x, z)+d(z, y)$, и метрического пространства $(X, d)$ были внедрены М. Фреше (см. [11]) и Ф. Хаусдорфом (см. [12]; см. историю вопроса в [2]) как частный случай топологического пространства (см. [13]). При этом неравенство треугольника $d(x, y) \leqslant d(x, z)+d(z, y)$ встречается уже у Евклида.

Бесконечные метрические пространства появляются обычно как обобщения естественной метрики $|x-y|$ на $\mathbb{R}$. Основными пространствами такого рода являются измеримые пространства (добавим меру) и банаховы пространства (добавим норму и полноту). Начиная с К. Менгера (1928) и Л. М. Блюменталя (1953), можно констатировать резкое повышение интереса ученых к конечным метрическим пространствам. Впрочем, широкое использование как конечных, так и бесконечных метрических пространств становится со второй половины XX в. трендом науки, потому что многие математические теории в ходе своего формирования стабилизируются именно на уровне метрического пространства (см. [14]). 
Этот процесс продолжается и сейчас. Метрики и расстояния давно и прочно стали важным инструментом исследований в самых разных областях математики и ее приложений, включая геометрию, теорию вероятностей, статистику, теорию кодирования, теорию графов, кластерный анализ, анализ данных, распознавание образов, теорию сетей, математическую инженерию, компьютерную графику, машинное зрение, астрономию, космологию, молекулярную биологию и многие другие отрасли науки. Создание наиболее удобных метрик стало центральной задачей для многих исследователей. Нередки случаи, когда одни и те же метрики появляются независимо друг от друга в совершенно разных сферах (расстояние между словами и расстояние (путь) эволюционного развития в биологии, расстояние Левенштейна в теории кодирования, расстояние Хэмминга с пропусками и др.).

В частности, к центральным объектам дискретной математики принадлежат метрический и разрезной конусы (см. [8]). Проблема максимального разреза, состоящая в нахождении для данного графа разреза максимального веса, является одной из наиболее значимых в комбинаторной оптимизации и имеет множество применений, в том числе в статистической механике.

Представляют значительный интерес и другие полиэдральные конструкции, связанные с конечными метриками, их аналогами и обобщениями: квазиметриками (несимметричный случай), $m$-метриками (многомерный случай) и т. д.

В данной статье мы рассматриваем вопросы, касающиеся конусов конечных квазиполуметрик, ориентированных разрезов и мультиразрезов и других ориентированных объектов, родственных метрикам, и соответствующую многомерную теорию, в том числе анализируем результаты машинных вычислений, полученные для таких конусов небольших размеров (см. [9]).

2. Метрики и их ориентированные и многомерные аналоги. В данном разделе мы вводим все основные определения, связанные с метриками и их ориентированными и многомерными аналогами.

Метрикой на $n$ точках называется функция $d:\{1, \ldots, n\}^{2} \rightarrow \mathbb{R}_{\geqslant 0}$, удовлетворяющая следующим условиям для всех $i, j, k \in\{1, \ldots, n\}$ :

(i) $d_{i i}=0$;

(ii) $d_{i j} \neq 0$ при $i \neq j$;

(iii) симметричность: $d_{i j}=d_{j i}$;

(iv) неравенства треугольника: $d_{i k} \leqslant d_{i j}+d_{j k}$.

При построении полиэдральных конструкций понятие метрики слишком ограничивает наши возможности, и значительно удобнее пользоваться его ослабленным аналогом - понятием полуметрики.

Полуметрикой на $n$ точках называется симметричная функция $d:\{1, \ldots, n\}^{2} \rightarrow \mathbb{R}_{\geqslant 0}$, удовлетворяющая следующим условиям для всех $i, j, k \in\{1, \ldots, n\}$ :

(i) $d_{i i}=0$;

(ii) неравенства треугольника: $d_{i k} \leqslant d_{i j}+d_{j k}$.

Другими словами, полуметрика - это метрика, допускающая нулевое расстояние между различными точками.

Одним из возможных обобщений (полу)метрик являются квази(полу)метрики, представляющие собой аналогичные, но несимметричные конструкции.

Квазиметрикой на $n$ точках называется функция $q:\{1, \ldots, n\}^{2} \rightarrow \mathbb{R}_{\geqslant 0}$, удовлетворяющая следующим условиям для всех $i, j, k \in\{1, \ldots, n\}$ :

(i) $q_{i i}=0$;

(ii) $q_{i j} \neq 0$ при $i \neq j$;

(iii) ориентированные неравенства треугольника: $q_{i k} \leqslant q_{i j}+q_{j k}$.

Другими словами, квазиметрика не всегда удовлетворяет условию симметричности $d_{i j}=d_{j i}$.

Квазиполуметрикой $q$ на $n$ точках называется функция $q:\{1, \ldots, n\}^{2} \rightarrow \mathbb{R}_{\geqslant 0}$, удовлетворяющая следующим условиям для всех $i, j, k \in\{1, \ldots, n\}$ :

(i) $q_{i i}=0$;

(ii) ориентированные неравенства треугольника: $q_{i k} \leqslant q_{i j}+q_{j k}$. 
Таким образом, квазиполуметрика - это «несимметричная полуметрика», или, что то же, «несимметричная метрика, допускающая нулевое значение на различных точках». Простейшим примером квазиполуметрики является измерение расстояний в городе, обладающим улицами с односторонним движением.

Квазиметрики были введены Хаусдорфом в [12], а их топология изучается с Вильсона (см. [15]). Конусы и многогранники квазиполуметрик изучались в $[2-4,6,9]$.

Для нашего исследования представляет интерес еще одно обобщение квазиполуметрик - так называемые $m$-(полу)метрики, являющиеся многомерными аналогами двумерного случая (полу)метрики.

$m$-Метрикой на $n$ точках называется функция $d:\{1, \ldots, n\}^{m+1} \rightarrow \mathbb{R}$, удовлетворяющая следующим условиям для всех $x_{1}, \ldots, x_{m+2} \in\{1, \ldots, n\}$ :

(i) неотрицательность: $d\left(x_{1}, \ldots, x_{m+1}\right) \geqslant 0$;

(ii) положительная определенность: $d\left(x_{1}, \ldots, x_{1}\right)=0$;

(iii) тотальная симметрия: $d\left(x_{1}, \ldots, x_{m+1}\right)=d\left(\pi\left(x_{1}\right), \ldots, \pi\left(x_{m+1}\right)\right)$ для любой перестановки $\pi$ множества $\left\{x_{1}, \ldots, x_{m+1}\right\}$

(iv) неравенства m-симплекса:

$$
d\left(x_{1}, \ldots, x_{m+1}\right) \leqslant \sum_{i=1}^{m+1} d\left(x_{1}, \ldots, x_{i-1}, x_{i+1}, \ldots, x_{m+2}\right) .
$$

$m$-Полуметрикой на $n$ точках называется функция $d:\{1, \ldots, n\}^{m+1} \rightarrow \mathbb{R}$, удовлетворяющая следующим условиям для всех $x_{1}, \ldots, x_{m+2} \in\{1, \ldots, n\}$ :

(i) неотрицательность: $d\left(x_{1}, \ldots, x_{m+1}\right) \geqslant 0$;

(ii) слабал положительная определенность: $d\left(x_{1}, \ldots, x_{m+1}\right)=0$ в случае, если $x_{1}, \ldots, x_{m+1}$ не являются попарно различными;

(iii) тотальная симметрия: $d\left(x_{1}, \ldots, x_{m+1}\right)=d\left(\pi\left(x_{1}\right), \ldots, \pi\left(x_{m+1}\right)\right)$ для любой перестановки $\pi$ множества $\left\{x_{1}, \ldots, x_{m+1}\right\}$;

(iv) неравенства т-симплекса:

$$
d\left(x_{1}, \ldots, x_{m+1}\right) \leqslant \sum_{i=1}^{m+1} d\left(x_{1}, \ldots, x_{i-1}, x_{i+1}, \ldots, x_{m+2}\right) .
$$

Заметим, что в некоторых источниках $m$-метрика получается из $m$-полуметрики при добавлении следующего условия: если $x_{1}, \ldots, x_{m}$ попарно различны, то $d\left(x_{1}, \ldots, x_{m}, x_{m+1}\right)>0$ для некоторого $x_{m+1}$.

Простейшим примером $m$-полуметрики является $m$-мерный объем в $\mathbb{R}^{n}, n \geqslant m . m$-Полуметрики были введены в [10].

\section{3. Примеры метрик, полуметрик и их ориентированных и многомерных аналогов.} Рассмотрим ряд базовых примеров.

3.1. Дискретная метрика. Для данного множества $X$, дискретная метрика-метрика на $X$, определенная формулой

$$
d(x, y)= \begin{cases}1, & \text { если } x \neq y \\ 0, & \text { иначе }\end{cases}
$$

3.2. Метрика Хаусдорфа. Пусть $(X, D)$ - конечное метрическое пространство и $X=X_{1} \cup \ldots \cup$ $X_{n}$ - разбиение $X$. Метрика Хаусдорфа-метрика $d$ на множестве $Y=\left\{X_{1}, \ldots, X_{n}\right\}$, определенная формулой

$$
d\left(X_{i}, X_{j}\right)=\max _{x \in X_{i}, y \in X_{j}} D(x, y) .
$$


3.3. Метрика симметрической разности. Для заданной антицепи множеств $Z=\{x, y, z, \ldots \mid$ $x \not \subset y$ для всех $x \neq y\}$, метрика симметрической разности-метрика на $Z$, определенная формулой

$$
d_{\triangle}(x, y)=|x \triangle y|
$$

Она равна числу элементов в симметрической разности

$$
x \triangle y=(x \backslash y) \cup(y \backslash x)
$$

множеств $x$ и $y$ из $Z$.

3.4. Дискретная квазиполуметрика. Пусть $X=\{1,2, \ldots, n\}$. Дискретная квазиполуметри$\kappa a$ - квазиполуметрика $q$ на $X$, определенная формулой

$$
q(x, y)= \begin{cases}1, & \text { если } x>y, \\ 0, & \text { иначе. }\end{cases}
$$

3.5. Квазиметрика Хаусдорба. Пусть $(X, D)$ - конечное метрическое пространство, и $X=$ $X_{1} \cup \ldots \cup X_{n}$ - разбиение $X$. Квазиметрика Хаусдорфа-квазиметрика $q$ на множестве $Y=$ $\left\{X_{1}, \ldots, X_{n}\right\}$, определенная формулой

$$
q\left(X_{i}, X_{j}\right):=\min _{x \in X_{i}} \max _{y \in X_{j}} D(x, y) .
$$

3.6. Квазисемиметрика асимметричной разности. Для заданной антицепи множеств $Z=$ $\{x, y, z, \ldots \mid x \not \subset y$ для всех $x \neq y\}$, метрика симметрической разности-метрика на $Z$, определенная формулой

$$
d_{\triangle}(x, y)=|x \triangle y|
$$

Она равна числу элементов в в асимметрической разности $x \backslash y$ множеств $x$ и $y$ из $Z$.

3.7. Дискретнал 2-метрика. Для заданного множества $X,|X| \geqslant 4$, дискретнал 2-метрика2 -метрика $d$ на $X$, определенная формулой

$$
d(x, y, z)= \begin{cases}0, & \text { если } x=y \text { или } y=z \text { или } x=z, \\ 1, & \text { иначе. }\end{cases}
$$

3.8. 2-Метрика Хаусдорфа. Пусть $(X, D)$ - конечное метрическое пространство и $X=X_{1} \cup \ldots \cup$ $X_{n}$ - разбиение $X$. Квазиметрика Хаусдорфа-квазиметрика $d$ на множестве $Y=\left\{X_{1}, \ldots, X_{n}\right\}$, определенная как

$$
d\left(X_{i}, X_{j}, X_{k}\right)=\frac{1}{3}\left(\max _{x \in X_{i}, y \in X_{j}} D(x, y)+\max _{x \in X_{i}, y \in X_{k}} D(x, y)+\max _{x \in X_{k}, y \in X_{j}} D(x, y)\right) .
$$

В общем случае $m$-метрика Хаусдорфа $d$ на $Y=\left\{X_{1}, \ldots, X_{n}\right\}$ определяется как

$$
\begin{aligned}
& d\left(X_{i_{1}}, X_{i_{2}}, \ldots, X_{i_{m+1}}\right)= \\
&=\frac{1}{m+1}\left(\max _{x \in X_{i_{1}}, y \in X_{i_{2}}} D(x, y)+\max _{x \in X_{i_{1}}, y \in X_{i_{3}}} D(x, y)+\ldots+\max _{x \in X_{i_{m}}, y \in X_{i_{m+1}}} D(x, y)\right) .
\end{aligned}
$$

3.9. 2-Полуметрика симметрической разности. Для заданной антицепи множеств $Z=$ $\{x, y, z, \ldots \mid x \not \subset y$ для всех $x \neq y\}, 2$-метрика симметрической разности-2-метрика $\rho$ на $Z$, определенная формулой

$$
\rho(x, y, z)=\frac{1}{2}(|x \triangle y|+|y \triangle z|+|x \triangle z|)
$$

это число элементов, которые принадлежат $x, y$ или $z$, но не всем трем множествам $x, y, z$.

Мы привели три группы примеров по три метрики в каждой группе: для классического симметрического двумерного случая, для ориентированного случая и для трехмерного (многомерного) случая. Заинтересованный читатель может построить свои примеры, например, связанные с метрикой пути связного графа. Эти построения, как и множество других интересных примеров, можно найти в [5]. 
4. Разрезы, мультиразрезы и их ориентированные и многомерные аналоги. Среди огромного числа различных полуметрик особую роль играют так называемые разрезы и мультиразрезы.

Разрезом на $n$ точках для множества $S \subseteq\{1, \ldots, n\}$ называется функция $\delta_{S}:\{1, \ldots, n\}^{2} \rightarrow \mathbb{R}$, определенная по закону

$$
\delta_{S}(i, j)= \begin{cases}1, & \text { если }|S \cap\{i, j\}|=1, \\ 0, & \text { иначе. }\end{cases}
$$

Легко проверить, что любой разрез является полуметрикой (не метрикой!). Мультиразрезом (точнее, $m$-мультиразрезом) на $n$ точках для разбиения $\bigcup_{i=1}^{m} S_{i}=\{1, \ldots, n\}$
называется функция $\delta_{S_{1}, \ldots, S_{m}}:\{1, \ldots, n\}^{2} \rightarrow \mathbb{R}$, определенная по закону

$$
\delta_{S}(i, j)= \begin{cases}1, & \text { если }|S \cap\{i, j\}|=1, \\ 0, & \text { иначе. }\end{cases}
$$

Очевидно, что 2-мультиразрезы совпадают с разрезами. Более того, любой мультиразрез можно представить в виде неотрицательной линейной комбинации разрезов:

$$
\delta_{S_{1}, \ldots, S_{m}}=\sum_{i=1}^{m} \delta_{S_{i}, \overline{S_{i}}} .
$$

Число всех мультиразрезов на $n$ точках представляет собой число Белла $B(n)$, т.е. число всех разбиений $n$-множества.

Ориентированные разрезы и мультиразрезы являются, в свою очередь, специальным случаем квазиполуметрик, несимметричным аналогом разрезов и мультиразрезов.

Ориентированным разрезом на $n$ точках для множества $S \subseteq\{1, \ldots, n\}$ называется функция $\delta_{S}^{\prime}:\{1, \ldots, n\}^{2} \rightarrow \mathbb{R}$, определенная по закону

$$
\delta_{S}^{\prime}(i, j)= \begin{cases}1, & \text { если } i \in S, j \notin S, \\ 0, & \text { иначе. }\end{cases}
$$

Легко проверить, что любой ориентированный разрез является квазиполуметрикой (не квазиметрикой!).

Ориентированным мультиразрезом (точнее, ориентированным т-мультиразрезом) для ориентированного разбиения $\bigcup_{i=1}^{m} S_{i}=\{1, \ldots, n\}$ множества $\{1, \ldots, n\}$ на $m$ частей $S_{1}, \ldots, S_{m}$ называется функция $\delta_{S_{1}, \ldots, S_{m}}^{\prime}:\{1, \ldots, n\}^{2} \rightarrow \mathbb{R}$, определенная по закону

$$
\delta_{S_{1}, \ldots, S_{m}}^{\prime}(i, j)= \begin{cases}1, & \text { если } i \in S_{a}, j \in S_{b} \text { и } a<b, \\ 0, & \text { иначе. }\end{cases}
$$

Очевидно, что ориентированные 2-мультиразрезы совпадают с ориентированными разрезами: $\delta_{S}^{\prime}=\delta_{S, \bar{S}}^{\prime}$. Однако в отличие от симметричного случая не каждый ориентированный мультиразрез (даже в простейшем случае $n=3$ ) можно представить в виде линейной комбинации ориентированных разрезов.

Число всех ориентированных мультиразрезов на $n$ точках представляет собой число Фубини (упорядоченное число Белла) $p^{\prime}(n)$, т.е. число всех упорядоченных разбиений множества $\{1, \ldots, n\}$; для перестановки $\pi=\left(a_{1}, \ldots, a_{n}\right) \in \operatorname{Sym}(n)$ оно равно $\sum_{\pi \in \operatorname{Sym}(n)} 2^{D(\pi)}$, где $D(\pi):=$ $\left|\left\{i \leqslant n: a_{i}>a_{i+1}\right\}\right|$.

$m$-Полуметрика разбиений - это многомерный аналог двумерного мультиразреза.

$m$-Полуметрикой разбиений для $(m+1)$-разбиения $S_{1}, \ldots, S_{m+1}$ множества $\{1, \ldots, n\}$, называется функция $\alpha_{S_{1}, \ldots, S_{m+1}}:\{1, \ldots, n\}^{m+1} \rightarrow \mathbb{R}$, определенная по закону

$$
\alpha_{S_{1}, \ldots, S_{m+1}}\left(i_{1}, \ldots, i_{m+1}\right)= \begin{cases}1, & \text { если } i_{1} \in S_{\alpha_{1}}, \ldots, i_{m+1} \in S_{\alpha_{m+1}}, \alpha_{k} \neq \alpha_{t} \text { для } k \neq t, \\ 0, & \text { если } i_{k}, i_{t} \in S_{\alpha} \text { для некоторого } k \neq t, 1 \leqslant \alpha \leqslant m+1 .\end{cases}
$$


Другими словами, величина $\alpha_{S_{1}, \ldots, S_{m+1}}\left(i_{1}, \ldots, i_{m+1}\right)$ равна 1 , если для всех $1 \leqslant j<l \leqslant m+1$ элементы $i_{j}$ и $i_{l}$ принадлежат разным подмножествам разбиения, и равна 0 во всех остальных случаях. Легко видеть, что $\alpha_{S_{1}, \ldots, S_{m+1}}$ является $m$-полуметрикой (не $m$-метрикой!), и что для $m=1$ она превращается в обычный разрез (см., например, [8]).

5. Конусы обобщенных дискретных метрических структур. Центральным разделом теории конечных полуметрик являются задачи комбинаторной оптимизации, решаемые полиэдральным методом, ведущим к построению соответствующих конусов и многогранников.

В данной работе мы ограничимся построением лишь конусов соответствующих конечных полуметрик и их ориентированных и многомерных аналогов. Однако исследование многогранников может быть проведено по той же схеме (см. [5]).

Метрическим конусом на $n$ точках $M E T_{n}$ называется множество всех полуметрик на $\{1, \ldots, n\}$.

Разрезным конусом (или конусом разрезов) на $n$ точках $C U T_{n}$ называется коническая оболочка (множество всех линейных комбинаций с неотрицательными коэффициентами) всех $2^{n-1}-1$ ненулевых разрезов на $\{1, \ldots, n\}$.

Следует заметить, что разрезный конус $C U T_{n}$ представляет собой множество $n$-точечных $l_{1}$ полуметрик, т.е. полуметрик, изометрически вложимых в пространство $l_{1}=\left(\mathbb{R}^{m},\|x-y\|_{1}\right)$ с $l_{1^{-}}$ нормой

$$
\|z\|_{1}=\sum_{i=1}^{m}\left|z_{i}\right|
$$

В пространстве меры величине $\|x-y\|_{1}$ соответствует $\mu(A \triangle B)$, где $A, B$ - множества, представляющие $x$ и $y$ (см. [8]).

Конусы $M E T_{n}$ и $C U T_{n}$ представляют собой полноразмерные конусы в $\mathbb{R}^{n(n-1) / 2}$. Поскольку любой разрез является полуметрикой, то выполняется очевидное включение $C U T_{n} \subseteq M E T_{n}$ с равенством лишь для $n=3,4$. Поскольку любой мультиразрез представляет собой неотрицательную линейную комбинацию разрезов, то говорить о конусе мультиразрезов смысла не имеет: коническая оболочка множества мультиразрезов является частью конуса $C U T_{n}$.

Полная группа симметрий конусов $C U T_{n}$ и $M E T_{n}$ представляет собой группу $\operatorname{Sym}(n)$ для $n \neq 4$ и группу $\operatorname{Sym}(4) \times \operatorname{Sym}(3)$ для $n=4$.

Квазиметрическим конусом на $n$ точках $Q M E T_{n}$ называется множество всех квазиполуметрик на $\{1, \ldots, n\}$.

Конусом ориентированных мультиразрезов на $n$ точках $O M C U T_{n}$ называется коническая оболочка всех $p^{\prime}(n)-1$ ненулевых ориентированных мультиразрезов на $\{1, \ldots, n\}$. Пользуясь формулой

$$
\delta_{S_{1}, \ldots, S_{m}}=\delta_{S_{1}, \ldots, S_{m}}^{\prime}+\delta_{S_{m}, \ldots, S_{1}}^{\prime}
$$

мы получаем, что $C U T_{n}=\left\{q+q^{T}: q \in O M C U T_{n}\right\}$.

Конусом ориентированных разрезов на $n$ точках $O C U T_{n}$ называется коническая оболочка всех $2^{n}-2$ ненулевых ориентированных разрезов на $\{1, \ldots, n\}$.

Поскольку в несимметричном случае ориентированный мультиразрез может и не быть линейной комбинацией ориентированных разрезов, то конусы $O M C U T_{n}$ и $O C U T_{n}$ не совпадают.

Конус $O C U T_{n}$ представляет собой множество всех $n$-точечных $l_{1}$-квазиполуметрик, т.е. квазиполуметрик, вложимых в квазиметрическое пространство $\left(\mathbb{R}^{m},\|x-y\|_{\text {оr.;1 }}\right)$ с ориентированной $l_{1}$-нормой

$$
\|z\|_{\text {or: } ; 1}=\sum_{i=1}^{m} \max \left(z_{i}, 0\right) .
$$

На пространстве меры величине $\|x-y\|_{\text {or:;1 }}$ соответствует $\mu(B \backslash A)$, где множества $B, A$ представляют $x, y$ (см. $[1,6])$.

Конусы $Q M E T_{n}$ и $O M C U T_{n}$ являются полномерными конусами в пространстве $\mathbb{R}^{n(n-1)}$.

Кроме очевидных строгих включений $O C U T_{n} \subset O M C U T_{n}$, мы имеем, с равенством только для $n=3$, включения $O M C U T_{n} \subseteq Q M E T_{n}$. 
В [7] показано, что конус $O C U T_{n}$ (определенный на множестве $\{1, \ldots, n\}$ ) есть проекция конуса $C U T_{n+1}$ (определенного на $\left.\{0,1, \ldots, n\}\right)$, на подпространство, ортогональное к $\delta_{\{0\}}$.

Экстремальные лучи конуса $Q M E T_{n}$ исследовались в [6], где было доказано, что они не являются симметричными и имеют по меньшей мере $n-1$ нулей, т.е. не могут быть расстояниями на ориентированном графе. Ориентированные мультиразрезы соответствуют экстремальным лучам конуса $Q M E T_{n}$. Кроме того, расщепление экстремального луча вновь дает экстремальный луч.

В [6] была представлена и таблица несмежности гиперграней конуса $Q M E T_{n}$; было сделано предположение о том, что во всех остальных случаях гиперграни смежны, откуда следует, что диаметр скелетона дуального конуса $Q M E T_{n}^{*}$ равен 2. Диаметр скелетона $Q M E T_{n}$ равен 3 для $n=4,5$.

Существует предположение, что диаметры $O C U T_{n}$ и $O M C U T_{n}$ равны 1 и 2 , соответственно. Более того, если $f \geqslant 0$ определяет гипергрань $O M C U T_{n}$, то нулевое расширение $f$ в $O M C U T_{n+1}$ останется гипергранью, как и в классическом случае конуса разрезов $C U T_{n}$.

Группа $\operatorname{Sym}(n)$ всех перестановок на $n$ точках является группой симметрий конусов $Q M E T_{n}$ и $O M C U T_{n}$. Но существует и другая симметрия, называемая реверселем. Для осуществления операции реверселя нужно поставить в соответствие каждому лучу $q$ луч $q^{T}$, определенный как $q_{i j}^{T}=q_{j i}$. (Другими словами, в матричных терминах реверсель соответствует транспонированию матриц.) Из этого факта следует, что группа $Z_{2} \times \operatorname{Sym}(n)$ также является группой симметрий для конусов $Q M E T_{n}$ и $O M C U T_{n}$. Мы предполагаем, что это их полная группа симметрий. Для конуса $O C U T_{n}$ этот факт доказан в [6].

Конусом m-полуметрик $H M E T_{n}^{m}$ называется множество всех $m$-полуметрик на $n$ точках.

Конусом m-полуметрик разбиений $\mathrm{HCUT}_{n}^{m}$ называется коническая оболочка всех $m$-полуметрик разбиений на $n$ точках.

Конусы $H M E T_{n}^{m}$ и $H C U T_{n}^{m}$ являются полномерными конусами в пространстве $\mathbb{R}_{n}^{E^{m+1}}$.

Поскольку каждая $m$-полуметрика разбиений является $m$-полуметрикой, получаем, что

$$
H C U T_{n}^{m} \subseteq H M E T_{n}^{m} \subseteq \mathbb{R}_{\geqslant 0}^{E_{n}^{m+1}} .
$$

Очевидно, что все грани конусов $H M E T_{n}^{m}$ и $H C U T_{n}^{m}$ сохраняются при любой перестановке вершин. Из этого следует, что любая такая перестановка порождает симметрию конусов $H M E T_{n}^{m}$ и $H C U T_{n}^{m}$, т.е. группа $\operatorname{Sym}(n)$ является группой симметрий указанных двух конусов. Применяя компьютерные подсчеты, мы проверили, что $\operatorname{Sym}(n)$ является полной группой симметрий для исследуемых конусов.

6. Таблица параметров рассматриваемых конусов. В таблице 1 представлена информация обо всех конусах, перечисленных выше, на малом числе вершин. Для каждого конуса мы приводим его размерность, число экстремальных лучей и число их орбит, число его максимальных граней и число их орбит, наконец, диаметры 1-скелетон-графа и ридж-графа.

7. Заключение. Помимо рассмотренных в статье несимметричных и многомерных аналогов классического симметричного случая метрик, разрезов, мультиразрезов и других родственных конструкций, интерес представляют их специальные частные случаи: взвешиваемые квазиметрики, частичные метрики, $m$-суперметрики и др. Построение и исследование соответствующих конусов и многогранников на малом числе точек можно провести по той же схеме (см. $[3,4])$.

\section{СПИСОК ЛИТЕРАТУРЫ}

1. Charikar M., Makarychev K., Makarychev V. Directed metrics and directed graph partitioning problem// in: Proc. 11 ACM-SIAM Symp. on Discrete Algorithms, 2006. - P. 51-60.

2. Deza M. M., Deza E. I. Encyclopedia of Distances. - Berlin: Springer-Verlag, 2014.

3. Deza M. M., Deza E. I. Cones of partial metrics// Contrib. Discr. Math. - 2011. - 6, № 1. - P. 26-47.

4. Deza M. M., Deza E. I., Vidali J. Cones of weighted and partial metrics// in: Proc. Int. Conf. on Algebra. - New Jersey: World Scientific, 2010. - P. 177-197.

5. Deza M. M., Dutour M., Deza E. I. Generalizations of Finite Metrics and Cuts. - World Scientific, 2016. 


\begin{tabular}{|c|c|c|c|c|}
\hline Конус & Размерность & \# экстр. лучей (орбит) & \# макс. граней орбит) & Диаметры \\
\hline$C U T_{3}=M E T_{3}$ & 3 & $3(1)$ & $3(1)$ & $1 ; 1$ \\
$C U T_{4}=M E T_{4}$ & 6 & $7(2)$ & $12(1)$ & $1 ; 2$ \\
$C U T_{5}$ & 10 & $15(2)$ & $40(2)$ & $1 ; 2$ \\
$M E T_{5}$ & 10 & $25(3)$ & $30(1)$ & $2 ; 2$ \\
$C U T_{6}$ & 15 & $31(3)$ & $210(4)$ & $1 ; 3$ \\
$M E T_{6}$ & 15 & $296(7)$ & $60(1)$ & $2 ; 2$ \\
$C U T_{7}$ & 21 & $63(3)$ & $38780(36)$ & $1 ; 3$ \\
$M E T_{7}$ & 21 & $55226(46)$ & $105(1)$ & $3 ; 2$ \\
$C U T_{8}$ & 28 & $127(4)$ & $49604520(2169)$ & $1 ; ?$ \\
$M E T_{8}$ & 28 & $119269588(3918)$ & $168(1)$ & $? ; 2$ \\
\hline$O M T_{3}=Q M E T_{3}$ & 6 & $12(2)$ & $12(2)$ & $2 ; 2$ \\
$O M C U T_{4}$ & 12 & $74(5)$ & $72(4)$ & $2 ; 2$ \\
$Q M E T_{4}$ & 12 & $164(10)$ & $36(2)$ & $3 ; 2$ \\
$O M C U T_{5}$ & 20 & $540(9)$ & $35320(194)$ & $2 ; 3$ \\
$Q M E T_{5}$ & 20 & $43590(229)$ & $80(2)$ & $3 ; 2$ \\
$O M C U T_{6}$ & 30 & $4682(19)$ & $150(2)$ & $2 ; ?$ \\
$Q M E T_{6}$ & 30 & $\geqslant 182403032(\geqslant 127779)$ & $120(4)$ & $2 ; 3$ \\
\hline$H C U T_{5}^{2}$ & 10 & $25(2)$ & $30(2)$ & $2 ; 2$ \\
$H M E T_{5}^{2}$ & 10 & $37(3)$ & $4065(16)$ & $2 ; 3$ \\
$H C U T_{6}^{3}$ & 15 & $65(2)$ & $45(2)$ & $3 ; 2$ \\
$H M E T_{6}^{3}$ & 15 & $287(5)$ & $674390(153)$ & $2 ; 3$ \\
$H C U T_{7}^{4}$ & 21 & $140(2)$ & $63(2)$ & $3 ; 2$ \\
$H M E T_{7}^{4}$ & 21 & $3692(8)$ & $84(2)$ & $2 ; ?$ \\
$H C U T_{8}^{5}$ & 28 & $266(2)$ & $108(2)$ & $3 ; 2$ \\
$H M E T_{8}^{5}$ & 28 & $55898(13)$ & $2095154(3086)$ & $? ; 2$ \\
$H M E T_{9}^{6}$ & 36 & $864174(20)$ & $80(2)$ & $3 ; ?$ \\
$H C U T_{6}^{2}$ & 20 & $90(3)$ & $175(2)$ & $? ; 2$ \\
$H M E T_{6}^{2}$ & 20 & $12492(41)$ & $140(2)$ & $? ; 2$ \\
$H M E T_{7}^{2}$ & 35 & $\geqslant 454191608(\geqslant 91836)$ & & \\
$H M E T_{7}^{3}$ & 35 & $\geqslant 551467967(\geqslant 110782)$ & & \\
\hline
\end{tabular}

Таблица 1. Параметры конусов на $n$ точках для малых $n$

6. Deza M. M., Dutour M., Panteleeva E. I. Small cones of oriented semi-metrics// Am. J. Math. Management Sci. - 2002. - 22, № 3-4. - P. 199-225.

7. Deza M. M., Grishukhin V. P., Deza E. I. Cones of weighted quasi-metrics, weighted quasi-hypermetrics and of oriented cuts// in: Mathematics of Distances and Applications. - Sofia: ITEA, 2012. - P. 31-53.

8. Deza M. M., Laurent M. Geometry of Cuts and Metrics. - Berlin: Springer-Verlag, 1997.

9. Deza M. M., Panteleeva E. I. Quasi-semi-metrics, oriented multi-cuts and related polyhedra// Eur. J. Combin. - 2000. - 21, № 6. - P. 777-795.

10. Deza M. M., Rosenberg I. G. n-Semimetrics// Eur. J. Combin. - 2000. - 21, № 6. - P. 797-806.

11. Fréchet M. Sur quelques points du calcul fonctionnel// Rend. Circ. Mat. Palermo. - 1906. - 22. - P. 1-74.

12. Hausdorff F. Grundzüge der Mengenlehre. - Leipzig, 1914.

13. Matthews S. G. Partial metric topology// Ann. New York Acad. Sci. - 1994. - 728. - P. 183-197.

14. Seda A. K. Quasi-metrics and the semantic of logic programs// Fundam. Inform. - 1997. - 29. - P. 97-117.

15. Wilson W. A. On quasi-metric spaces// Am. J. Math. - 1931. - 53. - P. 575-681.

Деза Елена Ивановна

Московский педагогический государственный университет

E-mail: elena.deza@gmail.com 